\title{
Thinning and disturbance growth in liquid films mobilized by continuous surfactant delivery
}

\author{
Benjamin J. Fischer and Sandra M. Troian ${ }^{\text {a) }}$ \\ Department of Chemical Engineering, Princeton University, Princeton, New Jersey 08544-5263
}

(Received 11 February 2003; accepted 10 September 2003; published 5 November 2003)

\begin{abstract}
A generalized linear stability analysis is applied to the case of a thin liquid film propelled to spread by a continuous supply of surfactant. The time-dependent base states for the film thickness and surfactant concentration give rise to a nonautonomous system describing disturbance propagation. As a first approximation, the nonautonomous operator is treated as time independent, thereby reducing the system of equations to a standard eigenvalue problem. For the range of parameters investigated, this modal approximation reveals a band of unstable modes corresponding to the growth of transverse, sinusoidal corrugations. A transient growth analysis of the fully time-dependent system, which requires the solution of an initial value problem, also signals the possibility of large disturbance growth. In both cases, significant amplification of infinitesimal disturbances can be traced to the region of the film most rapidly thinned by Marangoni stresses, which is characterized by large interfacial curvature and a sharp variation in shear stress. In contrast to previous models implementing a finite surfactant source that predict asymptotic stability, large transient growth and asymptotic instability are possible for the case of sustained surfactant release. (C) 2003 American Institute of Physics. [DOI: 10.1063/1.1623489]
\end{abstract}

\section{INTRODUCTION}

Previous investigations of disturbance growth in linearized models of spreading films coated by a finite, insoluble, and dilute surfactant monolayer have failed to predict the existence of asymptotically unstable modes, ${ }^{1-5}$ despite the prevalence of surfactant-induced instabilities in numerous experimental systems. ${ }^{6-9}$ These theoretical studies have shown that disturbances localized near the advancing monolayer front can undergo a brief period of rather large transient growth caused by significant variations in surfactant concentration and film curvature. This growth is strongly suppressed, however, once perturbations convect upstream toward the region of the film most sharply thinned by the initial Marangoni stresses following surfactant deposition. An earlier study ${ }^{4}$ examined the combined influence of van der Waals forces and Marangoni stresses on the stability of spreading films. Significant initial amplification of disturbances was obtained followed by a similar suppression, as observed in previous studies. Once the film was observed to thin beyond some critical height, however, disturbance amplification resumed and exceeded the values established at early times. Unfortunately, numerical instabilities associated with the onset of film rupture prevented exploration of the asymptotic behavior at long times.

It is unclear a priori which assumptions of the native model that predicates a finite, insoluble and dilute surfactant source are responsible for the lack of sustained transient growth or unstable modes at late times. In this paper, the assumption of a finite source, which leads to the diminution in time of the driving force responsible for spreading, is in-

${ }^{a)}$ Electronic mail: stroian@princeton.edu stead replaced by continuous surfactant delivery such that the total mass disbursed scales with time as $t^{1 / 2}$. This relation corresponds to the maximum rate of advance possible by an insoluble surfactant monolayer. ${ }^{10}$ By comparison with a finite source, the base-state film thickness and concentration profiles support a higher degree of film thinning and interfacial curvature for the same range of capillary and Peclet numbers.

The time-dependent base states for the film thickness and surfactant concentration give rise to a nonautonomous system of equations for disturbance propagation. As a first approximation, the nonautonomous operator is treated as time independent, thereby reducing the identification of the most unstable mode to a standard eigenvalue problem. For the range of parameters investigated, this modal approximation reveals a range of wave numbers corresponding to the growth of transverse, sinusoidal corrugations. A transient growth analysis of the fully time-dependent system, which requires the solution of an initial value problem, also signals the possibility of large disturbance growth. In both cases, the significant amplification of infinitesimal disturbances can be traced to the region of the film most rapidly thinned by Marangoni stresses, which is characterized by a large interfacial curvature and a sharp variation in shear stress. This analysis provides further evidence that sustained film thinning by continuous surfactant delivery, which causes a local bottleneck in surfactant transport along the air-liquid interface, leads to the ultimate destabilization of the spreading film by lateral film corrugations.

\section{GOVERNING EQUATIONS}

The spreading model consists of a Newtonian liquid with viscosity $\mu^{*}$, density $\rho^{*}$, and initial uniform thickness $h_{c}^{*}$ 


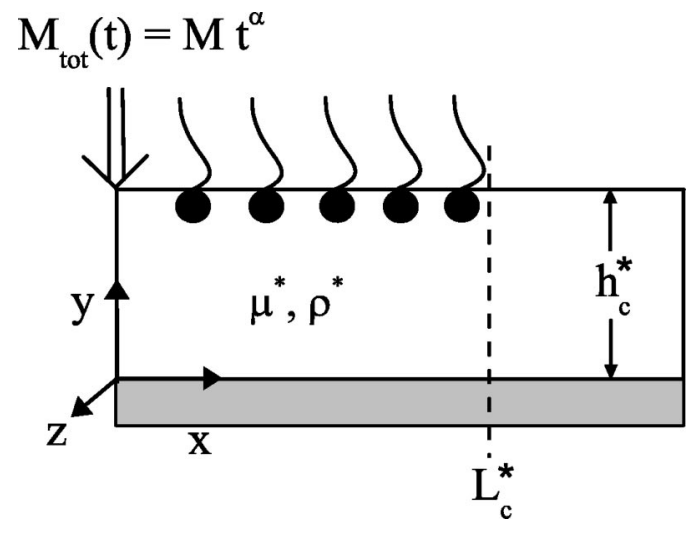

FIG. 1. Schematic diagram of the initial configuration at the outset of spreading. A Newtonian liquid characterized by a viscosity $\mu^{*}$, density $\rho^{*}$, and initial thickness $h_{c}^{*}$ is coated by an insoluble, dilute surfactant monolayer that initially extends a distance $L_{c}^{*}$. Surfactant is continually supplied at the origin to the spreading film such that the total surfactant mass increases as $M_{\text {tot }}(t)=M t^{\alpha}$, where $\alpha=1 / 2$.

contacted by a monolayer of insoluble surfactant. The initial (nonuniform) surfactant distribution extends a distance $L_{c}^{*}$, as shown in Fig. 1. The uncontaminated region is represented by a (higher) surface tension $\sigma_{0}^{*}$. The coated region is represented by a (lower) surface tension $\sigma_{m}^{*}$ corresponding to concentrations at or above the critical micelle concentration $\Gamma_{m}^{*}$. The maximum spreading pressure is therefore $\Pi^{*}$ $=\sigma_{0}^{*}-\sigma_{m}^{*}$. Utilizing the lubrication approximation and a linear equation of state relating surface tension to the surfactant surface concentration (the dilute approximation), the dimensionless evolution equations for the film thickness $H(x, z, t)$ and surfactant concentration $\Gamma(x, z, t)$ in the limit of negligible Bond number $\mathrm{Bo}=\rho^{*} g h_{c}^{* 2} / \Pi^{*}$ are given by $^{3,10}$

$$
\begin{aligned}
& \frac{\partial H}{\partial t}=\nabla \cdot\left[\frac{1}{2} H^{2} \nabla \Gamma-\frac{\mathcal{C}}{3} H^{3} \nabla^{3} H\right], \\
& \frac{\partial \Gamma}{\partial t}=\nabla \cdot\left[\Gamma H \nabla \Gamma-\frac{\mathcal{C}}{2} \Gamma H^{2} \nabla^{3} H+\frac{1}{\mathrm{Pe}_{s}} \nabla \Gamma\right] .
\end{aligned}
$$

Both the rectilinear streamwise $(x)$ and transverse $(z)$ coordinates have been scaled by $L_{c}^{*}$ and the film thickness and surface concentration are scaled by $h_{c}^{*}$ and $\Gamma_{m}^{*}$, respectively.

The two controlling dimensionless parameters are the inverse capillary number $\mathcal{C}=\varepsilon^{3} \sigma_{m}^{*} /\left(\mu^{*} u_{c}^{*}\right)=\varepsilon^{2} \sigma_{m}^{*} / \Pi^{*}$ and the surface Peclet number $\mathrm{Pe}_{s}=u_{c}^{*} L_{c}^{*} / \mathcal{D}_{s}^{*}$ where $\varepsilon$ $\equiv h_{c}^{*} / L_{c}^{*}$ and $\mathcal{D}_{s}^{*}$ is the surfactant surface diffusion coefficient. The characteristic flow speed is determined by the balance of Marangoni and viscous forces according to $u_{c}^{*}$ $=\varepsilon \Pi * / \mu^{*}$. The convective time scale is set by the ratio $L_{c}^{*} / u_{c}^{*}=\mu^{*} L_{c}^{* 2} / h_{c}^{*} \Pi^{*}$. Because this ratio scales as $L_{c}^{* 2}$, the initial extent of the monolayer, the characteristic time scales can be quite small, requiring longer integration times to establish significant film thinning and interfacial curvature.

\section{A. Base-state solutions for time-dependent source}

Jensen and Grotberg ${ }^{10}$ were the first to solve Eqs. (1) and (2) for the base-state profiles, $H_{0}(x, t)$ and $\Gamma_{0}(x, t)$, in a rectilinear geometry. They first considered the limit of pure Marangoni flow $\left(\mathcal{C}=1 / \mathrm{Pe}_{s}=0\right)$ to determine the similarity scalings inherent to the spreading process. Self-similar solutions of the form

$$
H_{0}(x, t)=h_{0}(\xi, t) \quad \text { and } \quad \Gamma_{0}(x, t)=\frac{g_{0}(\xi, t)}{t^{b}},
$$

were obtained in the transformed variable $\xi=x / t^{a}$. The total mass of surfactant disbursed for continuous surfactant delivery was described by

$$
M_{\mathrm{tot}}(t)=\int_{0}^{\infty} \Gamma_{0} d x=M t^{\alpha},
$$

where $M$ is time invariant. The advection of a finite amount of surfactant is represented by $\alpha=0$; time-dependent delivery corresponds to the case $\alpha>0$.

The constraints of self-similarity and boundedness of $\Gamma_{0}(x, t)$ imposes on Eqs. (1), (2), and (4) the algebraic relations

$$
a=\frac{1}{3}(1+\alpha) \quad \text { and } \quad b=\frac{1}{3}(1-2 \alpha),
$$

where $b \geqslant 0$. The limit $\alpha=1 / 2$ and $b=0$ corresponds to the maximum rate of surfactant propagation. This limit establishes the focus of this work.

Self-similarity can no longer be enforced with the inclusion of capillary forces and surface diffusion. Nonetheless, the transformations in Eq. (3) still provide a convenient coordinate system from which to view the evolution of the spreading film. For sufficiently small $\mathcal{C}$ and large $\mathrm{Pe}_{s}$, the solutions will approach a self-similar form. These variable transformations reduce Eqs. (1) and (2) to

$$
\begin{aligned}
t \frac{\partial h_{0}}{\partial t}= & a \xi h_{0 \xi}+\frac{1}{2}\left(h_{0}^{2} g_{0 \xi}\right)_{\xi}-\frac{\mathcal{C}}{3 t^{4 a-1}}\left(h_{0}^{3} h_{0 \xi \xi \xi}\right)_{\xi} \\
t \frac{\partial g_{0}}{\partial t}= & a \xi g_{0 \xi}+b g_{0}+\left(g_{0} h_{0} g_{0 \xi}\right)_{\xi}-\frac{\mathcal{C}}{2 t^{4 a-1}}\left(g_{0} h_{0}^{2} h_{0 \xi \xi \xi}\right)_{\xi} \\
& +\frac{t^{b}}{\mathrm{Pe}_{s}} g_{0 \xi \xi} .
\end{aligned}
$$

These coupled equations are subject to the following boundary conditions at $\xi=0$ and $\infty$ :

$$
\begin{aligned}
& h_{0 \xi}(0, t)=0, h_{0 \xi \xi \xi}(0, t)=0, \text { and } \\
& g_{0 \xi}(0, t)=-\frac{\alpha M}{h_{0}(0, t) g_{0}(0, t)+t^{b} / \mathrm{Pe}_{s}}, \\
& h_{0}(\infty, t)=1, h_{0 \xi}(\infty, t)=0, \text { and } g_{0}(\infty, t)=0 .
\end{aligned}
$$

The boundary conditions for $h_{0}$ in Eq. (8) specify film symmetry about the origin. The constraint on $g_{0 \xi}(0, t)$ in Eq. (8), 
which follows from the requirement of continuous surfactant delivery, is determined by integrating Eq. (7) by parts and utilizing the time invariance of $M=\int_{0}^{\infty} g_{0} d \xi$. The second set of boundary conditions in Eq. (9) imposes a quiescent and surfactant-free liquid film far downstream of the spreading monolayer. The initial conditions specified at $t=1$ are given by

$$
\begin{aligned}
& h_{0}(\xi, t=1)=1 \quad \text { and } \\
& g_{0}(\xi, t=1)=\left(\frac{1}{2} \xi_{w}^{2}-1 / \mathrm{Pe}_{s}\right) \exp \left(-\xi / \xi_{w}\right) .
\end{aligned}
$$

These initial distributions exactly satisfy the boundary condition for $g_{0 \xi}(0, t)$ specified in Eq. (8). ${ }^{10}$

Equations (6) and (7) were simultaneously solved using the method of lines ${ }^{11}$ that implements second-order centered differences for the spatial derivatives and a fully implicit Gear's method for the time derivative. ${ }^{12}$ The spatial domain was discretized with a nonuniform mesh, concentrating more points near the origin and in the vicinity of $\xi=0.1$, where the film undergoes severe and rapid film thinning. Figure 2 shows the evolution in time of the base-state variables $h_{0}$, $g_{0}$, and $g_{0 \xi}$ at $t=1,10,10^{2}, 10^{3}, 10^{4}, 10^{5}$, and $10^{6}$ for $\alpha$ $=1 / 2, \xi_{w}=1 / 4, \mathrm{Pe}_{s}=1000$, and $\mathcal{C}=10^{-4}$. As the grid spacing near $\xi=0.1$ decreased, the solutions converged to those shown Fig. 2. For the particular solutions shown, a spatial domain of length 1.5 was discretized with 920 grid points, which created grid spacings of $\Delta \xi=10^{-4}$ near $\xi=0.1$ and $\Delta \xi=5 \times 10^{-3}$ in the downstream region of flow.

As evident in Fig. 2, the nonuniform distribution of surfactant causes liquid flow toward the uncontaminated region. The Marangoni forces pull liquid and surfactant toward the region of higher surface tension, resulting in a large ridge at the advancing front and a sharply thinned region from $\xi$ $=0$ to $\xi=0.15$. The thinning behind the monolayer front slows the advection of surfactant past this region-this, coupled with the continuous delivery of surfactant, causes a rise in $g_{0}(\xi=0, t)$. The surfactant bottleneck established near $\xi=0.10$ also produces a local spike in $g_{0 \xi}(\xi=0.10, t)$. At long times, the film thickness and concentration profiles collapse to a universal curve whose shape is strongly governed by the self-similar solutions for purely Marangoni flow. ${ }^{10}$

Continuous surfactant delivery with $\alpha=1 / 2$ generates substantial film thinning for $t \geqslant 10^{4}$. Of interest to this work is the effect of this rapid film depletion and the subsequent spike in $g_{0 \xi}(\xi=0.10, t)$ on film stability. The linear stability analysis focuses on the amplification of disturbances at these longer time scales. Shorter times can be examined by adjusting the parameter $\xi_{w}$, which controls the initial mass of surfactant deposited on the liquid film.

\section{B. Disturbance equations for time-dependent source}

Disturbances in the the height, $\widetilde{H}(x, z, t)$, and concentration field, $\widetilde{G}(x, z, t)$, which are periodic in the direction transverse to the flow $(z)$ and characterized by wave number $Q$, are considered. In the transformed coordinate system,

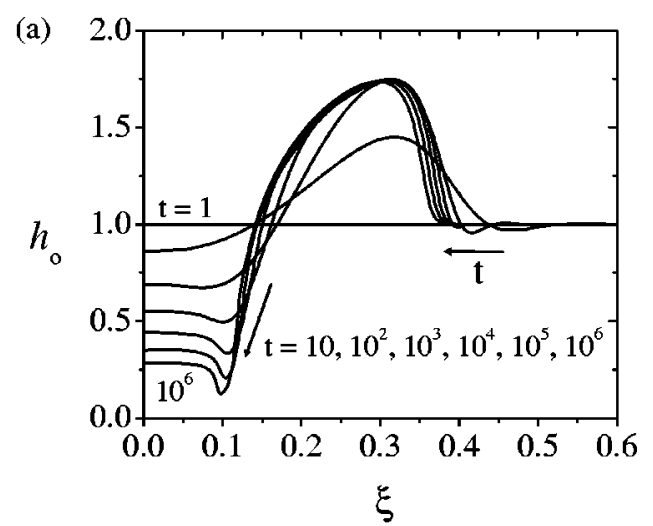

(b)

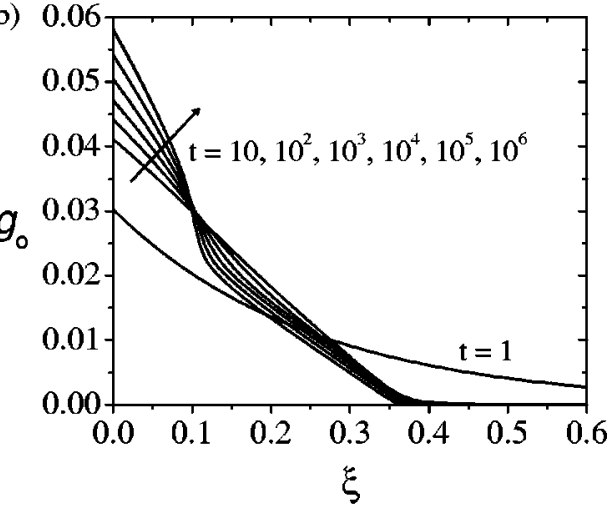

(c)

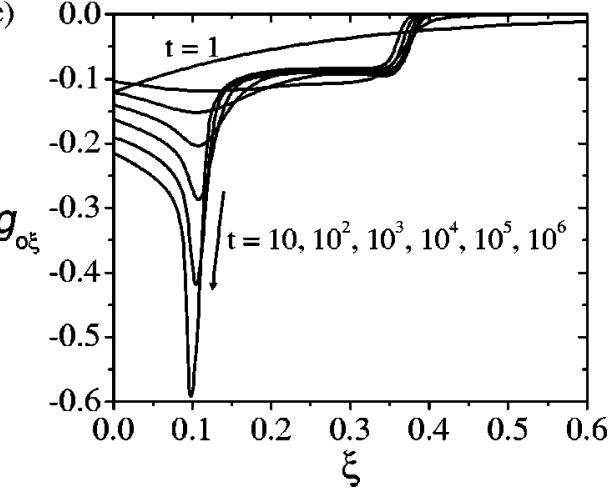

FIG. 2. Numerical solutions of the unperturbed (base-state) profiles for times $t=1,10,10^{2}, 10^{3}, 10^{4}, 10^{5}$, and $10^{6}, \alpha=1 / 2, \mathrm{Pe}_{s}=1000$ and $\mathcal{C}$ $=10^{-4}$ : (a) film thickness, $h_{0}(\xi, t)$, (b) surface surfactant concentration, $g_{0}(\xi, t)$, and (c) surfactant concentration gradient $g_{0 \xi}(\xi, t)$.

$$
\widetilde{H}(x, z, t)=\Psi(\xi, t) e^{i Q z} \quad \text { and } \quad \widetilde{G}(x, z, t)=\frac{\Phi(\xi, t)}{t^{b}} e^{i Q z} .
$$

Linearization of the governing evolution equations leads to the coupled system for $\Psi(\xi, t)$ and $\Phi(\xi, t)$ :

$$
\begin{aligned}
t \frac{\partial \Psi}{\partial t}= & a \xi \Psi_{\xi}+\frac{1}{2}\left(h_{0}^{2} \Phi_{\xi}+2 h_{0} g_{0 \xi} \Psi\right)_{\xi}-\frac{\left(Q t^{a}\right)^{2}}{2} h_{0}^{2} \Phi \\
& -\frac{\mathcal{C}}{3 t^{4 a-1}}\left[\left(h_{0}^{3} \Psi_{\xi \xi \xi}+3 h_{0}^{2} h_{0 \xi \xi \xi} \Psi\right)_{\xi}\right. \\
& \left.-\left(Q t^{a}\right)^{2}\left(3 h_{0}^{2} h_{0 \xi} \Psi_{\xi}+2 h_{0}^{3} \Psi_{\xi \xi}\right)+\left(Q t^{a}\right)^{4} h_{0}^{3} \Psi\right],
\end{aligned}
$$




$$
\begin{aligned}
t \frac{\partial \Phi}{\partial t}= & a \xi \Phi_{\xi}+b \Phi+\left(g_{0} g_{0 \xi} \Psi+h_{0} g_{0 \xi} \Phi+h_{0} g_{0} \Phi_{\xi}\right)_{\xi} \\
& -\left(Q t^{a}\right)^{2} h_{0} g_{0} \Phi-\frac{\mathcal{C}}{2 t^{4 a-1}}\left[\left(g_{0} h_{0}^{2} \Psi_{\xi \xi \xi}\right.\right. \\
& \left.+2 g_{0} h_{0} h_{0 \xi \xi \xi} \Phi\right)_{\xi}-\left(Q t^{a}\right)^{2}\left(\left(g_{0} h_{0}^{2}\right)_{\xi} \Psi_{\xi}\right. \\
& \left.\left.+2 g_{0} h_{0}^{2} \Psi_{\xi \xi}\right)+\left(Q t^{a}\right)^{4} g_{0} h_{0}^{2} \Psi\right]+\frac{t^{b}}{\mathrm{Pe}_{s}}\left[\Phi_{\xi \xi}\right. \\
& \left.-\left(Q t^{a}\right)^{2} \Phi\right] .
\end{aligned}
$$

The decoupling of the $\xi$ and $z$ coordinates in Eqs. (12) and (13) leads to a linearized set of equations simply expressed in matrix form, according to

$$
t \frac{\partial \mathbf{u}}{\partial t}=\mathrm{A}(t) \cdot \mathbf{u},
$$

where $\mathbf{u}=[\Psi, \Phi]$ is the disturbance vector and $\mathrm{A}(t)$ the matrix propagator. The temporal dependence of $\mathrm{A}$ arises through the explicit time dependence introduced by the coordinate transformation as well as the implicit time dependence of the base-state variables $h_{0}(\xi, t)$ and $g_{0}(\xi, t)$. The boundary conditions applied to these disturbance equations at $\xi=0$ and $\xi=\infty$ are given by

$$
\begin{aligned}
& \Psi_{\xi}(0, t)=0, \quad \Psi_{\xi \xi \xi}(0, t)=0, \quad \text { and } \quad \Phi_{\xi}(0, t)=0, \\
& \Psi_{\xi}(\infty, t)=0, \quad \Psi_{\xi \xi \xi}(\infty, t)=0, \quad \text { and } \quad \Phi(\infty, t)=0 .
\end{aligned}
$$

Only the slope but not the amplitude of $\Psi(\xi, t)$ is required to vanish as $\xi \rightarrow \infty$ in order to allow for bounded spatial oscillations far from the spreading front, where the film has a uniform thickness of unity. An eigenvalue analysis that implements this type of condition will capture the continuous spectrum corresponding to capillary wave disturbances on the flat film. ${ }^{13,14}$ Inclusion of the continuous spectrum is essential for understanding the complete eigenspectrum and has been shown to be critical in other unbounded freesurface flows such as the thin-film flow over topography. ${ }^{14}$

\section{EIGENSPECTRUM FOR STATIONARY DISTURBANCE OPERATOR}

The nonautonomous nature of $\mathrm{A}(t)$ in Eq. (14) prevents computation of the most unstable modes by a standard eigenvalue analysis. The base-state solutions shown in Figs. 2(a) and 2(b), however, approach self-similar form for $t>10^{4}$, which allows for the possibility of investigating the stability of a "quasisteady" system. As a first approximation, then, the full time-dependent matrix is converted to stationary form $\mathrm{A}\left(t_{c}\right)$, where $t_{c}$ lies within the range where the base states start collapsing onto a common shape. All terms on the right-hand sides of Eqs. (12) and (13) are therefore evaluated as constants and the identification of the most unstable modes simply requires a calculation of the leading eigenvalues of $\mathrm{A}\left(t_{c}\right)$. This approximation can be interpreted as a probe of the "instantaneous" stability of the spreading film.

Because of the factor $t$ that precedes the partial derivative in the term $t(\partial \mathbf{u} / \partial t)$ of Eq. (14), the disturbances are assumed to evolve in algebraic fashion according to

$$
\Psi(\xi, t)=\psi(\xi) t^{\beta} \quad \text { and } \quad \Phi(\xi, t)=\phi(\xi) t^{\beta},
$$

which reduces Eq. (14) to

$$
\beta \hat{\mathbf{u}}=\mathrm{A}\left(t_{c}\right) \cdot \hat{\mathbf{u}},
$$

where $\hat{\mathbf{u}}=[\psi, \phi]$ represents the disturbance vector. Algebraic growth is assumed in order to remove the time dependence on the left-hand side of Eq. (14), but exponential growth can be recovered with the transformation $t \rightarrow \ln t$. The growth rate $\beta$ for a given disturbance wave number $Q$ is determined by solving Eq. (18). These modes determine the eigenspectrum of $\mathrm{A}\left(t_{c}\right)$.

For unbounded domains $(0<\xi<\infty)$, there exist additional solutions corresponding to the continuous spectrum. ${ }^{13,14}$ Unlike discrete modes, which are required to decay as $\xi \rightarrow \infty$, the continuous modes allow for bounded spatial oscillations like capillary waves far from the spreading front. An estimate of the growth rate of these modes can be obtained by considering the limiting behavior of Eqs. (12) and (13) as $\xi \rightarrow \infty$, where $h_{0}(\xi \rightarrow \infty, t)=1, g_{0}(\xi \rightarrow \infty, t)=0$, $\Psi_{\xi}(\xi \rightarrow \infty, t)=0$, and $\Phi(\xi \rightarrow \infty, t)=0$. The substitution of these limiting values reduces Eq. (12) to

$$
\beta \psi=-\frac{\mathcal{C}}{3 t_{c}^{4 a-1}}\left(\psi_{\xi \xi \xi \xi}-2 Q^{2} t_{c}^{2 a} \psi_{\xi \xi}+Q^{4} t_{c}^{4 a} \psi\right) .
$$

Sinusoidal disturbances of the form $\psi \sim e^{i s \xi}$ yield the dispersion relation, $\beta(Q)$, governing the locus of the essential spectrum,

$$
\beta=-\frac{1}{3} \mathcal{C} t_{c}^{1-4 a} s^{4}-\frac{2}{3} \mathcal{C} t_{c}^{1-2 a} Q^{2} s^{2}-\frac{1}{3} \mathcal{C} t_{c} Q^{4} .
$$

Within the stationary approximation, this result confirms that capillary wave disturbances at the domain boundary $\xi \rightarrow \infty$ are stable for all wave numbers. The maximum growth rate $\beta$ occurs in the limit $s=0$, which defines a wave of constant amplitude $\psi(\xi)$ whose growth rate is given by

$$
\beta=-\frac{1}{3} \mathcal{C} t_{c} Q^{4} .
$$

The eigenvalues and eigenvectors of Eq. (18), which correspond to the disturbance growth rates $\beta$ and shapes $\psi(\xi)$ and $\phi(\xi)$, respectively, were calculated with the sparse eigenvalue solver EIGS in MATLAB. The derivatives of the disturbance functions were discretized on the same mesh used for the base flow calculation with second-order centered difference formulas. Figure 3 shows how the disturbance growth rate $\beta$ is affected by $t_{c}$ for wave numbers $0 \leqslant Q \leqslant 3$. . For times $t_{c}<5 \times 10^{4}$, the system is stable to disturbances of any wave number. If the system of equations is instead "frozen" at a later time $t_{c} \geqslant 5 \times 10^{4}$, then unstable modes develop.

Figures 4 and 5 depict the growth rates and disturbance amplitudes, $\psi(\xi)$ and $\phi(\xi)$, for two choices of $t_{c}$ corresponding to "stable" and "unstable" configurations. For $t_{c}=10^{4}$, the dispersion curve shown in Fig. 4 clearly shows that the system is stable to disturbances of wave number $0 \leqslant Q$ $\leqslant 3.0$. No unstable modes are evident beyond this range in wave number. At low wave numbers, the largest eigenvalue is dominated by the continuous mode solution specified in Eq. (21). This mode corresponds to a bounded capillary 


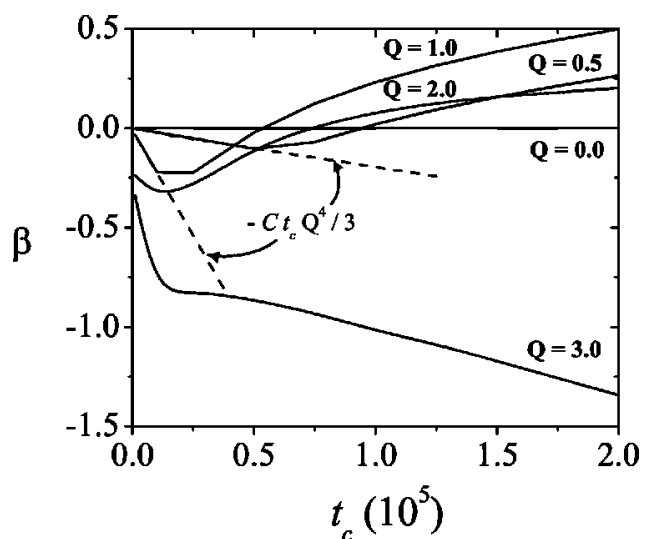

FIG. 3. Numerical solution of the instantaneous growth rate, $\beta\left(Q, t_{c}\right)$, for disturbances with wave numbers in the range $0.0 \leqslant Q \leqslant 3$. . The time $t_{c}$ denotes the time at which the linear operator is held stationary for an evaluation of the associated eigenspectrum.

wave and is designated by the abbreviation "CW" in Fig. 4(b). In contrast to the discrete modes, the continuous modes $\psi$ and $\phi$ display some structure in the upstream region where the film has undergone significant thinning, as well as in the downstream region at the advancing ridge and beyond. For wave numbers greater than $Q \approx 0.9$, the largest eigenvalue instead corresponds to solutions of the discrete eigenspectrum. The associated eigenfunctions only show a structure in the thinned region near $\xi=0.1$ and vanish everywhere else, as seen in Figs. 4(b) and 4(c).

Once the base-state solutions evolve past $t=5 \times 10^{4}$, the film thinning near $\xi=0.1$ becomes significant. When the relevant operator is frozen at $t_{c}=10^{5}$, the dispersion relation shown in Fig. 5 signals the presence of unstable modes for $0.5 \leqslant Q \leqslant 2.1$. The maximum growth rate occurs at $Q \approx 1.3$, which slightly decreases as $t_{c}$ increases. As with the solutions for $t_{c}=10^{4}$, the largest eigenvalue for low wave numbers is controlled by the continuous spectrum. An example of this bounded capillary wave is shown in Fig. 5(b) and is designated by the abbreviation "CW." As before, the continuous modes $\psi$ and $\phi$ display a clear structure in the upstream region, where the film has undergone significant thinning, as well as in the downstream region at the advancing ridge and beyond. For wave numbers larger than $Q \approx 0.4$, the largest eigenvalue is instead controlled by the discrete spectrum. The associated eigenfunctions, $\psi(\xi)$ and $\phi(\xi)$, peak in the vicinity of $\xi=0.1$, and vanish everywhere else.

\section{AMPLIFICATION PROFILES FOR TIME-DEPENDENT DISTURBANCE OPERATOR}

There is no reference frame that renders the base-state profiles shown in Fig. 2 time independent because the capillary and diffusion terms preclude self-similar solutions. The nonautonomous nature of $\mathrm{A}(t)$ in Eq. (14) therefore requires that the linear stability be investigated through a generalized stability analysis. Farrell and Ioannou ${ }^{15,16}$ suggest that for nonautonomous systems the first Lyapunov exponent, which reduces to the spectral abscissa in autonomous systems, should be monitored. This calculation, however, is computa- (a)

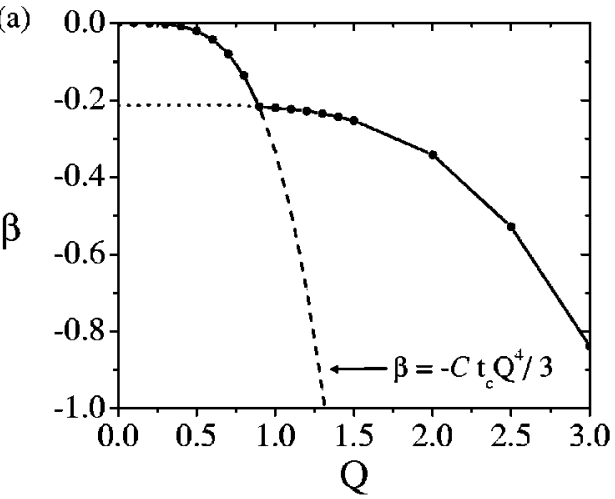

(b)
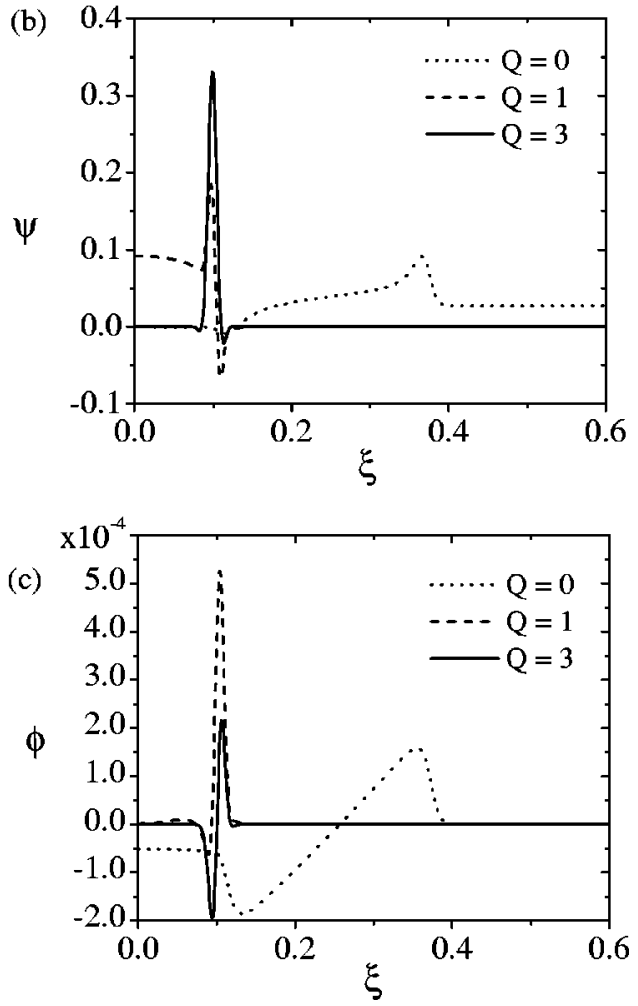

FIG. 4. Numerical solutions of disturbance (a) growth rates, (b) film shapes, $\psi(\xi)$, and (c) concentration profiles, $\phi(\xi)$, for $t_{c}=10^{4}$. The dashed line in (a) traces out the growth rates associated with the continuous modes (capillary waves), as given by Eq. (21). The dotted line in (a) represents a subdominant mode corresponding to the discrete spectrum. Other parameter values are $\alpha=1 / 2, \mathrm{Pe}_{s}=1000$, and $\mathcal{C}=10^{-4}$.

tionally difficult because of the large number of grid points needed to resolve the base-state solutions shown in Fig. 2.

An alternative analysis reduces the system to an initial value problem and the results are therefore dependent on the shape, location, and time at which disturbances are applied. Because the base states are time dependent, disturbance growth must be measured relative to these evolving base states. ${ }^{5,17,18}$ For instance, a system in which the base states decay in time appears unstable when perturbations either amplify or decay at a slower rate than the base states. Similarly, a system appears stable when disturbances decay or amplify at a slower rate than the amplifying base states. This intensification or dissipation of the disturbances relative to the base states is quantified by the amplification ratio 
(a)

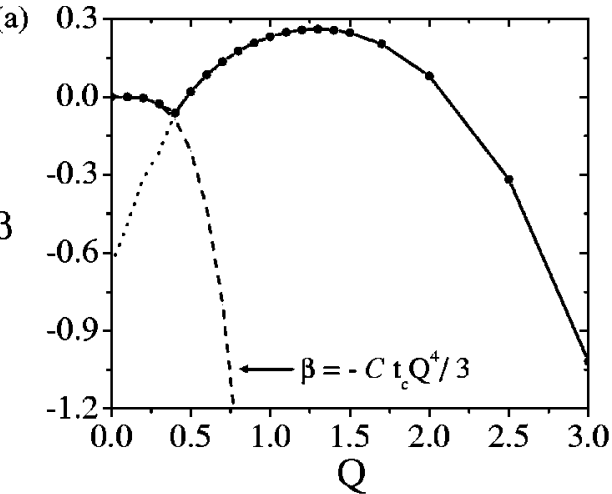

(b)
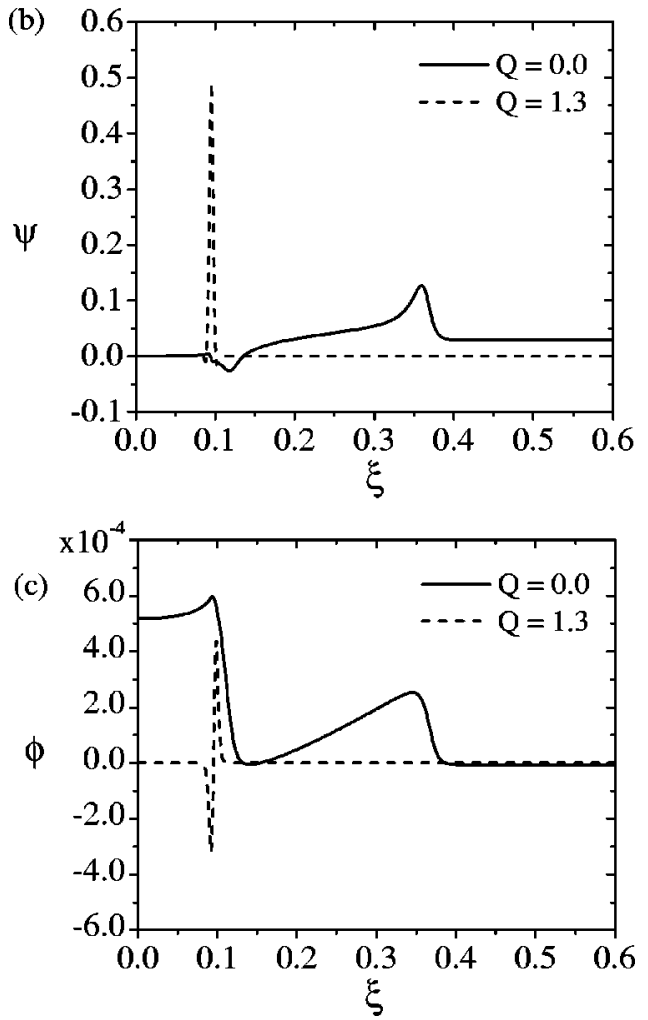

FIG. 5. Numerical solutions of disturbance (a) growth rates, (b) film shapes, $\psi(\xi)$, and (c) concentration profiles, $\phi(\xi)$, for $t_{c}=10^{5}$. The dashed line in (a) traces out the growth rates associated with the continuous modes (capillary waves), as given by Eq. (21). The dotted line in (a) represents a subdominant mode corresponding to the discrete spectrum. Other parameter values are $\alpha=1 / 2, \mathrm{Pe}_{s}=1000$, and $\mathcal{C}=10^{-4}$.

$$
R(t)=\left[\frac{E_{d}(t)}{E_{d}\left(t_{i}\right)}\right] /\left[\frac{E_{b}(t)}{E_{b}\left(t_{i}\right)}\right],
$$

where the energy of the base state, $E_{b}(t)$, and applied disturbances, $E_{d}(t)$, are normalized by the corresponding values at a reference time $t_{i}$. While a calculation of the first Lyapunov vector and exponent provides a more rigorous definition of stability for nonautonomous systems, ${ }^{16}$ the approach outlined here helps isolate the features of the basestate profiles that enhance perturbation growth.

The amplification ratio defined by Eq. (22) requires specification of the measure for monitoring the production or dissipation of energy. Because the film thickness and surfactant concentration are coupled variables that both determine the spreading velocity, it is physically appealing to consider a single measure of amplification and growth associated with the actual kinetic energy contained in the flow. The heightaveraged kinetic energy of the flow per unit wavelength in the transverse direction, $\lambda=2 \pi / Q$, is given by

$$
\begin{aligned}
& E_{b} \equiv \frac{1}{2 \lambda} \int_{0}^{\lambda} \int_{0}^{\infty}\left|\left\langle\mathbf{v}_{\mathbf{0}}\right\rangle\right|^{2} d \xi d z \quad \text { and } \\
& E_{d} \equiv \frac{1}{2 \lambda} \int_{0}^{\lambda} \int_{0}^{\infty}|\langle\widetilde{\mathbf{v}}\rangle|^{2} d \xi d z,
\end{aligned}
$$

where the subscripts $b$ and $d$ denote the energy of the basestate and disturbance, respectively. The magnitude of the base-state velocity vector is given by $\left|\left\langle\mathbf{v}_{\mathbf{0}}\right\rangle\right|$ and that of the disturbance velocity field by $|\langle\widetilde{\mathbf{v}}\rangle|$. All height-averaged quantities are denoted by angular brackets. The base-state velocity fields in the streamwise $\left(u_{0}\right)$ and transverse directions $\left(w_{0}\right)$ are given by

$$
\begin{aligned}
& \left\langle u_{0}\right\rangle=-\frac{1}{2 t^{a+b}} h_{0} g_{0 \xi}+\frac{\mathcal{C}}{3 t^{3 a}} h_{0}^{2} h_{0 \xi \xi \xi}, \\
& \left\langle w_{0}\right\rangle=0 .
\end{aligned}
$$

(The vertical component is negligible within the lubrication approximation.) The disturbance velocity fields are given by

$$
\begin{aligned}
\langle\tilde{u}\rangle= & {\left[-\frac{1}{2 t^{a+b}}\left(h_{0} \Phi_{\xi}+g_{0 \xi} \Psi\right)+\frac{\mathcal{C}}{3 t^{3 a}}\left(2 h_{0} h_{0 \xi \xi \xi} \Psi\right.\right.} \\
& \left.\left.+h_{0}^{2} \Psi_{\xi \xi \xi}-Q^{2} t^{2 a} h_{0}^{2} \Psi_{\xi}\right)\right] e^{i Q z}, \\
\langle\widetilde{w}\rangle= & {\left[-\frac{1}{2 t^{b}} h_{0} \Phi+\frac{\mathcal{C}}{3 t^{2 a}} h_{0}^{2}\left(\Psi_{\xi \xi}-Q^{2} t^{2 a} \Psi\right)\right] i Q e^{i Q z} . }
\end{aligned}
$$

To make a comparison with the stationary results obtained in Sec. III, it is useful to evaluate the amplification ratios, $R\left(t, t_{i}\right)$, at times $t_{i}=t_{c}=10^{4}$ and $10^{5}$. To fortify this comparison, the initial conditions for the disturbance functions are chosen to be the discrete modes (with the largest eigenvalue) obtained from the modal analysis. At $t=t_{i}$, these perturbations were applied to the system and Eqs. (6), (7), (12), and (13) were simultaneously solved using the method of lines. ${ }^{11,12}$ The results that follow were found to converge on a nonuniform mesh consisting of 920 points with grid spacings of $\Delta \xi=10^{-4}$ near $\xi=0.1$ and $\Delta \xi=5$ $\times 10^{-3}$ in the downstream region of flow.

Figure 6 shows the energy amplification ratio $R(t)$ for perturbations of four different wave numbers with $t_{i}=10^{4}$ and $10^{5}$. As evident, the system undergoes an initial stabilizing response when $t_{i}=10^{4}$. This response is not entirely unexpected because the earlier modal calculation demonstrated stability for $t_{c}=10^{4}$. With increasing $t$, the smaller wave number disturbances amplify while those with $Q \geqslant 3.0$ undergo rapid decay. By the time $t=10^{6}, R(t)$ increases by nearly a factor of 300 for the wave function $Q=1$. This behavior is consistent with the earlier modal analysis where $\beta>0$ for a restricted set of wave numbers. Additionally, the critical time at which the transient growth intensifies is simi- 


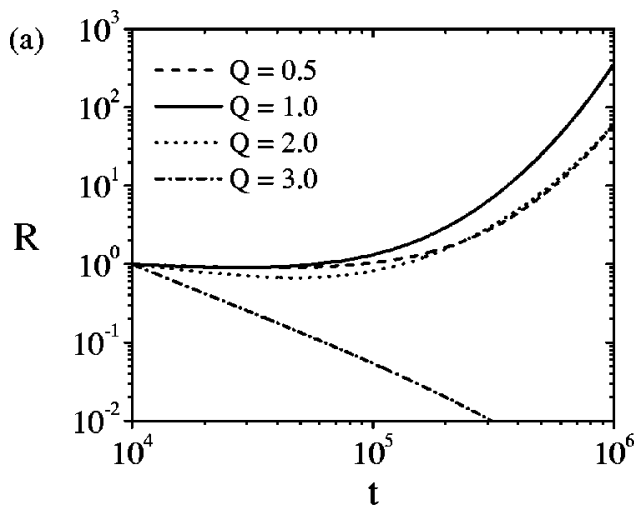

(b)

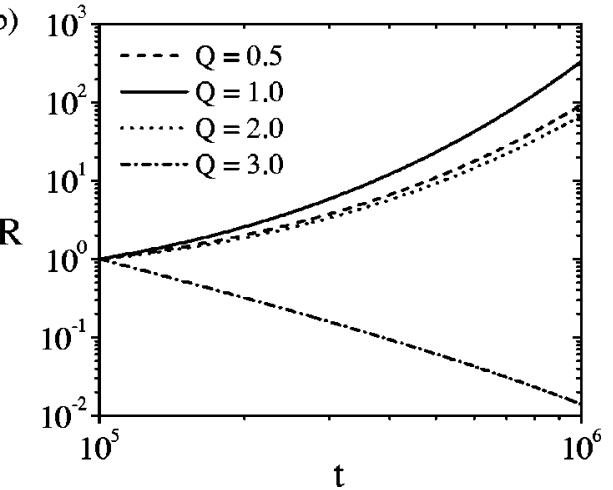

FIG. 6. Numerical solutions of the normalized (energy) amplification ratios for disturbances with wave numbers $0.5 \leqslant Q \leqslant 3.0$ applied to the spreading film at times (a) $t_{i}=10^{4}$ and (b) $t_{i}=10^{5}$. Other parameter values are $\alpha$ $=1 / 2, \mathrm{Pe}_{s}=1000$, and $\mathcal{C}=10^{-4}$

lar to the critical time for unstable growth obtained with the stationary approximation (see Fig. 3); in both cases, perturbations grow when $t \approx 5 \times 10^{4}$.

When disturbances are applied to the spreading film at a later time $t_{i}=10^{5}$, there is no initial stabilizing response but a rapid increase in $R(t)$, as evident in Fig. 6(b). By $t_{i}$ $=10^{5}$, the base-state solutions for the film thickness shown in Fig. 2(a) indicate a significant dip near $\xi=0.1$, and the modal analysis predicts instability for a selected band of wave numbers. Figure 7 depicts the growth of disturbances with $Q=1$ applied at $t_{i}=10^{5}$. As time elapses, the disturbances remain localized in the upstream region of the flow where the film significantly thins. Furthermore, the amplitudes of $\Psi$ and $\Phi$ increase in time, coinciding with the increase of $R$ in Fig. 6(b). In Fig. 7(c) is shown the superposition $h(\xi, z, t)=h_{0}(\xi, t)+\delta \Psi(\xi, t) \sin (Q z)$ of the base state $h_{0}(\xi, t)$ and disturbance profile $\Psi(\xi, t)$ for $t=5 \times 10^{5}$ and $Q=1$ with $\delta=0.05$. This total height profile $h(\xi, z, t)$ is only shown on the domain $0 \leqslant \xi \leqslant 0.15$ in order to accentuate the unstable structures located in the thinned region behind the advancing front. Disturbances applied prior to $t_{i}=5 \times 10^{4}$ are found to exhibit similar behavior as those applied at $t_{i}$ $=10^{4}$. Specifically, the perturbations undergo an initial stabilizing response coinciding with the local decrease in $R(t)$. For times greater than $t \approx 5 \times 10^{4}$, the system develops substantial amplification of modes localized to the region near $\xi=0.1$, which is expected to produce deep transverse corrugations in the spreading film.
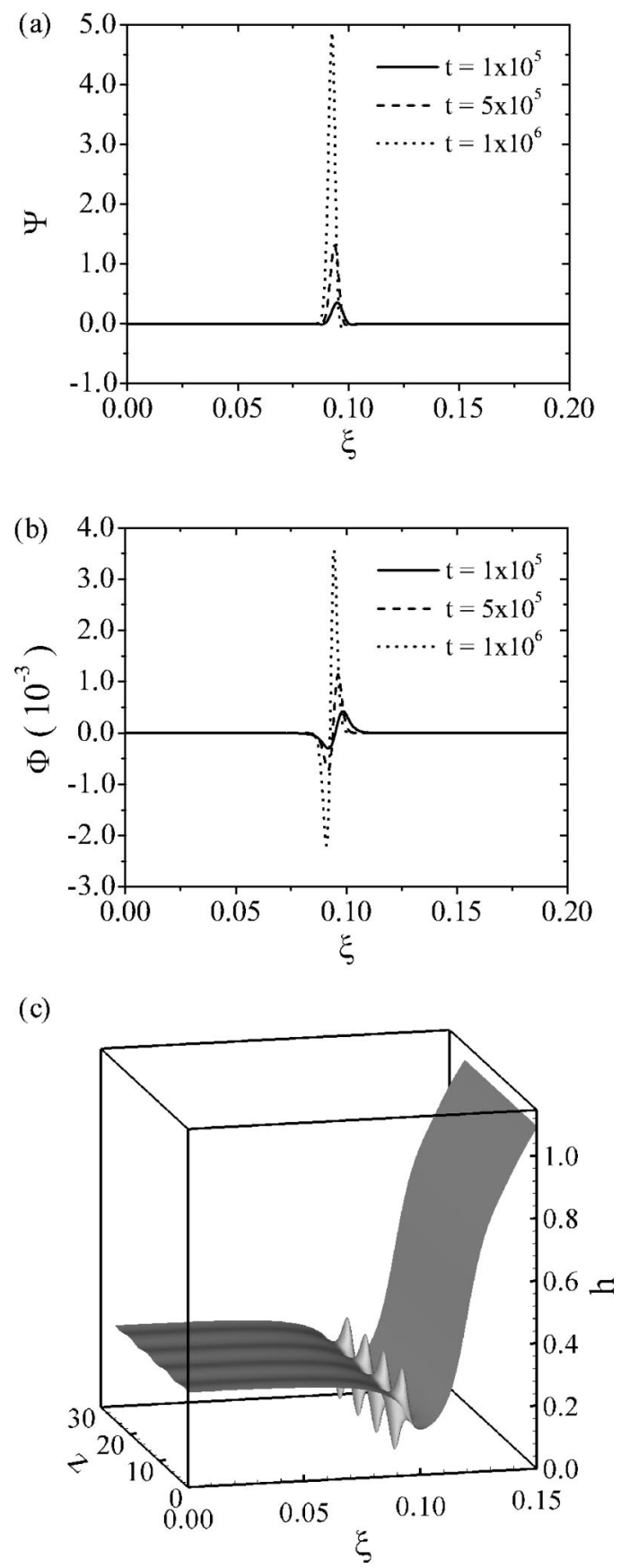

FIG. 7. The evolution of (a) $\Psi(\xi, t)$ and (b) $\Phi(\xi, t)$ for $Q=1$ and $t_{i}$ $=10^{5}$. (c) The total height profile $h$ at $t=5 \times 10^{5}$ for $Q=1$. Other parameter values are given by $\alpha=1 / 2, \mathrm{Pe}_{s}=1000$, and $\mathcal{C}=10^{-4}$.

\section{DISCUSSION}

Both the stationary (modal) approximation, as well as the fully time-dependent linear stability analysis, indicate that the source of instability lies in the upstream region of the film, which is characterized by a deepening furrow. This dip leads to a bottleneck effect that slows the advection of surfactant past this region. The continuous delivery of surfactant at the origin exacerbates this effect. Ahead of the furrow, surfactant is pulled toward the uncontaminated region of the film by a forward Marangoni flow. At the furrow, there de- 
velops a stagnation point in $g_{0}$ accompanied by a significant variation in $g_{0 \xi}$. As the furrow thins, this effect intensifies and leads to instability.

In previous work on Marangoni spreading propelled by a finite surfactant monolayer, a full transient analysis revealed that the region of high curvature and rapid change in $g_{0 \xi}$ at the leading edge of the advancing ridge was responsible for significant disturbance amplification ${ }^{5}$ at early times. In this study, disturbances were applied to different regions of the spreading film. As these disturbances traveled upstream, they underwent rapid amplification while advecting past the thickened portion of the film at the capillary ridge. The system was found to restabilize, however, as these disturbances migrated farther upstream. Although the disturbances also localized to the thinnest region of the film, where their speed sharply decreases, it was found that the transient amplification could not be sustained and the amplification ratio $R(t)$ rapidly decayed thereafter.

In this present study, which allows continuous surfactant delivery, the spreading film not only develops a region of high curvature and rapid change in $g_{0 \xi}$ at the advancing capillary ridge, but also in the upstream portion of the flow, as shown in Fig. 2. When disturbances finally localize to this region, there is apparently enough surfactant behind the furrow to destabilize the flow. As the furrow continues to thin, the maximum shear stress, which is proportional to $\left(-g_{0 \xi}\right)$, increases in time, leading to continued growth in $R(t)$.

\section{CONCLUSION}

In this study we examine film thinning and disturbance growth in thin liquid films mobilized to spread by a continuous delivery of an insoluble, dilute surfactant monolayer. The total mass of disbursed surfactant scales with time as $t^{1 / 2}$. The associated rate of delivery corresponds to the maximum rate of advance possible by an insoluble surfactant monolayer spreading on a thin viscous film. While the base-state solutions for the film thickness and surfactant concentration in the pure Marangoni-driven limit allow for self-similar collapse under an appropriate variable transformation, the inclusion of capillary forces and surface diffusion precludes fully time-independent base states. The linearized operator associated with this set of equations is therefore nonautonomous and a straightforward eigenvalue analysis to determine the most unstable modes is not possible.

Both an eigenvalue analysis on the stationary nonautonomous operator and a full transient study demonstrated perturbation growth once the base-state film profile sufficiently thinned and the concentration gradient in this region was highly curved. Disturbances were shown to preferentially localize in this thin region behind the advancing front and those characterized by a selected band of wave numbers grew. These results provide evidence that sustained film thinning by continuous surfactant delivery, which causes a local bottleneck in surfactant transport along the air-liquid interface, leads to the ultimate destabilization of the spreading film by lateral film corrugations.
In order to examine the linear stability of the full timedependent model of a spreading film continuously supplied with surfactant, initial conditions on the spatial structure of the perturbations were required. There are many such choices possible. This study has focused on the set of initial conditions derived from the eigenvectors of the associated stationary matrix. Simulations with Gaussian-type perturbations (such as those used in linear stability studies on the spreading of a finite amount of surfactant ${ }^{2,3,5}$ ) demonstrate similar perturbative growth; namely, disturbances significantly amplify once the upstream region of the base-state develops sufficient film thinning and a strong variation in the concentration gradient. Further studies, however, should examine the effect of varying the initial conditions on the spatial and temporal evolution of the disturbance fields.

A comparison of these results to experimental observations is difficult due to the lack of quantitative data available on the formation of fingering patterns. Parameter estimates in the literature for $h_{c}^{*}, \Pi^{*}, \mu^{*}$, and $L_{c}^{*}$ (Refs. 7-9) suggest (dimensional) characteristic time scales ranging from $10^{-3}-10 \mathrm{~s}$. The perturbative growth observed in this study would therefore suggest dimensional onset times for fingering ranging from $10-10^{5} \mathrm{~s}$. The onset times for instability, however, also strongly depend on $\xi_{w}$ in Eq. (10) because this parameter determines the initial mass of surfactant. Additionally, the large parameter space for the theoretical computations hinders a direct comparison to experimental measurements. This work, however, clearly shows that unstable structures preferentially grow in the thinned film region behind the advancing front, as experimentally observed. ${ }^{6-8}$

Film thinning and destabilization by purely attractive van Waals interactions are well known. ${ }^{19-21}$ It will be interesting to determine if the inclusion of an additional force that promotes film thinning in a nonlinear fashion will drive instability at even earlier times in the spreading process. Similarly, replacing the dilute approximation by a more realistic constitutive equation for the surface tension as a function of concentration should also strongly influence the onset time for large transient growth.

\section{ACKNOWLEDGMENTS}

Financial support from the National Science Foundation (CTS-0088774 and DMR-0213706), Unilever Research U.S., and the U.S. Army (ARDEC) is gratefully acknowledged.

${ }^{1}$ O. K. Matar and S. M. Troian, "Linear stability analysis of an insoluble surfactant monolayer spreading on a thin liquid film," Phys. Fluids 9, 3645 (1997).

${ }^{2}$ O. K. Matar and S. M. Troian, "Growth of non-modal transient structures during the spreading of surfactant coated films," Phys. Fluids 10, 1234 (1998).

${ }^{3}$ O. K. Matar and S. M. Troian, "The development of transient fingering patterns during the spreading of surfactant coated films," Phys. Fluids 11, 3232 (1999).

${ }^{4}$ O. K. Matar and S. M. Troian, "Spreading of a surfactant monolayer on a thin liquid film: Onset and evolution of digitated structures," Chaos 9, 141 (1999).

${ }^{5}$ B. J. Fischer and S. M. Troian, "Growth and decay of localized disturbances on a surfactant-coated spreading film," Phys. Rev. E 67, 016309 (2003).

${ }^{6}$ B. J. Fischer, A. A. Darhuber, and S. M. Troian, "Streamlets and branching 
dynamics in surfactant driven flow," Phys. Fluids 13, S11 (2001).

${ }^{7}$ M. Cachile, M. Schneemilch, A. Hamraoui, and A. M. Cazabat, "Films driven by surface tension gradients," Adv. Colloid Interface Sci. 96, 59 (2002).

${ }^{8}$ S. M. Troian, X. L. Wu, and S. A. Safran, "Fingering instability in thin wetting films," Phys. Rev. Lett. 62, 1496 (1989).

${ }^{9}$ B. Frank and S. Garoff, "Origins of complex motion of advancing surfactant solutions," Langmuir 11, 87 (1995).

${ }^{10}$ O. E. Jensen and J. B. Grotberg, "Insoluble surfactant spreading on a thin viscous film: Shock evolution and rupture," J. Fluid Mech. 240, 259 (1992).

${ }^{11}$ W. E. Schiesser, The Numerical Method of Lines (Academic, San Diego, 1991).

${ }^{12}$ A. C. Hindmarsh, in Scientific Computing, edited by R. S. Stepleman (North-Holland, Amsterdam, 1983), p. 55.

${ }^{13} \mathrm{Y}$. Ye and H. C. Chang, "A spectral theory for fingering on a prewetted plane," Phys. Fluids 11, 2494 (1999).
${ }^{14}$ S. Kalliadasis and G. M. Homsy, "Stability of free-surface thin-film flows over topography," J. Fluid Mech. 448, 387 (2001).

${ }^{15}$ B. F. Farrell and P. J. Ioannou, "Generalized stability theory. Part I: Autonomous operators," J. Atmos. Sci. 53, 2025 (1996).

${ }^{16}$ B. F. Farrell and P. J. Ioannou, "Generalized stability theory. Part II: Nonautonomous operators,” J. Atmos. Sci. 53, 2041 (1996).

${ }^{17}$ S. F. Shen, "Some considerations of the laminar stability of incompressible time-dependent basic flows," J. Aerosp. Sci. 28, 397 (1961).

${ }^{18}$ J. M. Davis, B. J. Fischer, and S. M. Troian, in Interfacial Fluid Dynamics in Physiochemical Phenomena, edited by R. Narayanan (Springer-Verlag, New York, 2003).

${ }^{19}$ E. Ruckenstein and R. K. Jain, "Spontaneous rupture of thin liquid films," J. Chem. Soc., Faraday Trans. 70, 132 (1974).

${ }^{20}$ M. P. Ida and M. J. Miksis, "The dynamics of thin films I: General theory," SIAM (Soc. Ind. Appl. Math.) J. Appl. Math. 58, 456 (1998).

${ }^{21}$ A. Oron, "Three-dimensional nonlinear dynamics of thin liquid films," Phys. Rev. Lett. 85, 2108 (2000). 